MS38-P03

\section{Understanding elastically driven cooperativity in molecular photomagnetic materials}

Alix Volte ${ }^{1}$, Céline Mariette ${ }^{1}$, Marie-Laure Boillot ${ }^{2}$, Marco

Cammarata $^{1}$, Giovanni Azzolina ${ }^{1}$, Matteo Levantino ${ }^{3}$, Roman

Bertoni ${ }^{1}$, Maciej Lorenc ${ }^{1}$

1. Institut de Physique de Rennes, UMR 6251, Rennes, France

2. Institut de Chimie Moléculaire et des Matériaux d'Orsay, Orsay, France

3. ESRF, Grenoble, France

email: alix.volte@univ-rennes1.fr

Materials can be controlled via physical parameters like pressure or temperature. With the advent of ultrashort lasers pulses (typically femtosecond), light excitation has been added to the panel of available techniques for materials control. Two of the major aspects in so-called photo-induced phase transitions are:

- reversibility, meaning the ability to switch back and forth between two states,

- efficiency, corresponding to the ratio of the amount of transformed material on the quantity of provided light.

One possible approach to amplify the photoresponse is based on the material elastic properties. This was demonstrated for a light-sensitive and volume-changing Spin Crossover (SCO) material [1], meaning, a molecular crystal in which the unit cell is built with SCO molecule(s). In this molecule, the ligand field result in a splitting of the $3 \mathrm{~d}$ levels of the metal ion (FeIII) into low energy and high energy ones. The population of high energy levels (at high temperature) changes the spin state and is accompanied by strong changes in Fe-ligand distances (and thus unit cell volume).

The sudden generation (via a laser pulse) of a high enough fraction of photo-excited molecules (switched from Low spin to High spin state) creates local negative pressure. This drives lattice expansion that can induce additional switching of neighboring molecules through positive feedback effect. This cooperative effect is associated as expected with a well-defined threshold mechanism. We can actually see (insert in Fig.) that the fraction of transformed molecules after photoexcitation decreases monotonically for low excitation fraction, while it increases non-linearly in the nanosecond time scale for higher ones.

To unambiguously discriminate between spin state conversion and structural changes, we performed Xray time-resolved diffraction study at synchrotron ESRF. The diffraction patterns measured from ps to $\mu$ s time delays on nanocrystals powder films give a direct signature of the ultrafast volume expansion. Quantitative analysis (Rietveld) of the powder spectra at ps time scale allows going deeper into the understanding of the cooperative aspects of the photo-induced spin conversion in these molecular materials.
References:

[1] R.Bertoni, M.Lorenc, H.Cailleau, A.Tissot, J.Laisney, M. Boillot, L. Stoleriu, A. Stancu, C.Enachescu \& E.Collet. Elastically driven cooperative response of a molecular material impacted by a laser pulse,Nature Materials 15, 606-610, (2016)

[2] R. Bertoni, M. Lorenc, T. Graber, R. Henning, K. Moffat, J.-F. Létard, and E. Collet, Cooperative elastic switching vs laser heating in [Fe(phen)2(NCS)2] spin-crossover crystals excited by a laser pulse. Cryst.Eng.Comm. 18, 7269-7275 (2016)

Keywords: $x$-ray powder diffraction, photo-induced transitions, time-resolved transitions 